\title{
O professor, as condições de trabalho e os efeitos sobre sua saúde*
}

\author{
Sandra Maria Gasparini \\ Sandhi Maria Barreto \\ Ada Ávila Assunção \\ Universidade Federal de Minas Gerais
}

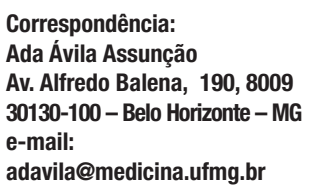

*Agradecemos à Profa Dalila Andrade Oliveira pelo esforço na coordenação do Grupo de Estudos sobre Trabalho Docente - GESTRADO. Este Grupo, apoiado pelo CNPq e pela FAPEMIG, está ligado ao Núcleo de Estudos sobre Trabalho e Educação [NETE] da Faculdade de Educação/UFMG, que proporciona 0 ambiente necessário para as reflexões tratadas neste artigo.

\section{Resumo}

0 estudo das relações entre o processo de trabalho docente, as reais condições sob as quais ele se desenvolve e o possível adoecimento físico e mental dos professores constituem um desafio e uma necessidade para se entender o processo saúde-doença do trabalhador docente e se buscar as possiveis associações com o afastamento do trabalho por motivo de saúde.

Este artigo apresenta o perfil dos afastamentos do trabalho por motivos de saúde de uma população de profissionais da educação. Buscando elementos na literatura disponível, aventa a hipótese de que as condições de trabalho nas escolas podem gerar sobreesforço dos docentes na realização de suas tarefas.

Foram analisados os dados apresentados no Relatório preparado pela Gerência de Saúde do Servidor e Perícia Médica (GSPM) da Prefeitura Municipal de Belo Horizonte, Minas Gerais, relativos aos afastamentos do trabalho de funcionários da Secretaria $\mathrm{Mu}-$ nicipal de Educação, de abril de 2001 a maio de 2003. Os afastamentos foram indicados pelos atestados médicos fornecidos pela própria instituição.

Os dados obtidos, embora não permitissem discriminar o número de professores envolvidos, possibilitaram o conhecimento do número de afastamentos entre os professores, sendo que os transtornos psíquicos ficaram em primeiro lugar entre os diagnósticos que provocaram os afastamentos.

\section{Palavras-chave}

Trabalho docente - Professor - Saúde do trabalhador - Saúde mental e trabalho. 


\title{
The teacher, working conditions and their effects on his health*
}

\author{
Sandra Maria Gasparini \\ Sandhi Maria Barreto \\ Ada Ávila Assunção \\ Federal University of Minas Gerais
}

Correspondence:

Ada Ávila Assunção

Av. Alfredo Balena, n. 190, 8009

30130-100 - Belo Horizonte - MG

e-mail: adavila@medicina.ufmg.br

* We would like to thank Professor Dalila Andrade Oliveira for her work at the coordination of the Study Group on Teacher Work - GESTRADO. This group, sponsored by $\mathrm{CNPq}$ and FAPEMIG, is associated to the Center for Studies on Work and Education (NETE) of the Faculty of Education UFMG, which provides the adequate environment for the reflections presented in this article.

\begin{abstract}
The study of the relations between the teaching work process, the real conditions under which it is developed, and the possible physical and mental ailment of teachers constitutes a challenge and a necessity to understand the health-sickness process of teachers, and to seek new associations with sick leaves.

This article presents the sick leave profile of a population of professionals in education. Drawing from the available literature, the study raises the hypothesis that the working conditions at schools may be leading to teachers' overload when carrying out their duties.

The study analyzed data presented in the Report prepared by the Worker Health Administration and Medical Examination Office (GSPM) of the City of Belo Horizonte, Minas Gerais, corresponding to sick leaves by employees of the Municipal Secretary for Education between April 2001 and May 2003. The sick leaves were recommended in medical reports issued by the GSPM itself.

The data obtained, although not allowing to ascertain the total number of teachers involved, made is possible to know the number of sick leaves among teachers. Psychiatric disorders were top of the list among the diagnostics that resulted in sick leaves.
\end{abstract}

\section{Keywords}

Teacher work - Teacher - Worker health - Work and mental health. 
Avaliando o contexto das políticas que visam à educação para todos, este artigo apresenta a hipótese da defasagem das condições de trabalho em face das metas traçadas e efetivamente alcançadas, as quais acabam gerando sobreesforço dos docentes na realização de suas tarefas.

A Organização Internacional do Trabalho definiu as condições de trabalho para os professores ao reconhecer o lugar central que estes ocupam na sociedade, uma vez que são os responsáveis pelo preparo do cidadão para a vida (0IT, 1984). Tais condições buscam basicamente atingir a meta de um ensino eficaz.

As transformações sociais, as reformas educacionais e os modelos pedagógicos derivados das condições de trabalho dos professores provocaram mudanças na profissão docente, estimulando a formulação de políticas por parte do Estado. De acordo com Souza et al. (2003), até os anos de 1960, a maior parte dos trabalhadores do ensino gozavam de uma relativa segurança material, de emprego estável e de um certo prestígio social. Já a partir dos anos de 1970, a expansão das demandas da população por proteção social provocou o crescimento do funcionalismo e dos serviços públicos gratuitos, entre eles a educação.

$\mathrm{Na}$ atualidade, o papel do professor extrapolou a mediação do processo de conhecimento do aluno, o que era comumente esperado. Ampliou-se a missão do profissional para além da sala de aula, a fim de garantir uma articulação entre a escola e a comunidade. 0 professor, além de ensinar, deve participar da gestão e do planejamento escolares, o que significa uma dedicação mais ampla, a qual se estende às famílias e à comunidade.

Embora o sucesso da educação dependa do perfil do professor, a administração escolar não fornece os meios pedagógicos necessários à realização das tarefas, cada vez mais complexas. Os professores são compelidos a buscar, então, por seus próprios meios, formas de requalificação que se traduzem em aumento não reconhecido e não remunerado da jornada de trabalho (Teixeira, 2001; Barreto e Leher, 2003; Oliveira, 2003).
É pertinente defender que o sistema escolar transfere ao profissional a responsabilidade de cobrir as lacunas existentes na instituição, a qual estabelece mecanismos rígidos e redundantes de avaliação e contrata um efetivo insuficiente, entre outros. A título de exemplo, em 2002, o IV Congresso Nacional de Educação registrou o déficit nacional de professores em educação básica no Brasil, pois eram necessários mais 836731 para a educação infantil, 167706 para o ensino fundamental e 215 mil para o ensino médio (Souza et al., 2003). Os dados do Ministério da Educação, já em 2004, esclarecem que, somente no ensino médio, faltam na rede, para citar apenas um dos casos de insuficiência de efetivo, 23,5 mil professores de física (MEC/INEP, 2004).

Como obter sucesso nos objetivos estabelecidos para o ensino num período de pouca oferta de vagas, com salas de aula repletas de crianças e adolescentes? Quais seriam os efeitos para o professor, se, no espaço da produção do ensino, não lhe são garantidas as condições adequadas para atingir as metas que orientam as reformas educacionais recentes? Sob essas condições, o único elemento de ajuste é o trabalhador, que, com seus investimentos pessoais, procura auxiliar o aluno carente comprando material escolar e restringindo o seu tempo supostamente livre para criar estratégias pedagógicas que compensem a ausência de laboratórios, de salas de informática e de bibliotecas minimamente estruturadas (Noronha, 2001).

Na última década, o trabalho docente tornou-se, por demanda do sindicalismo, tema de vários estudos e de investigações, incentivando a formação de grupos e de redes de pesquisadores organizados para esse fim. Souza et al. (2003, p. 106) resumem sete projetos de pesquisa com resultados consistentes e abrangentes que dão visibilidade, nos anos de 1990, às precárias condições do trabalho docente e mostram sua associação com sintomas mórbidos e a elevada prevalência de afastamentos por motivos de doença na categoria. 
As condições de trabalho, ou seja, as circunstâncias sob as quais os docentes mobilizam as suas capacidades físicas, cognitivas e afetivas para atingir os objetivos da produção escolar podem gerar sobreesforço ou hipersolicitação de suas funções psicofisiológicas. Se não há tempo para a recuperação, são desencadeados ou precipitados os sintomas clínicos que explicariam os índices de afastamento do trabalho por transtornos mentais, conforme descrito no que segue.

\section{Metodologia}

Esta pesquisa documental foi realizada tendo como texto base o Relatório da Prefeitura Municipal de Belo Horizonte (Belo Horizonte, 2003) elaborado juntamente com o Sindicato Único dos Trabalhadores em Educação em Minas Gerais - sind-UTE, representante da categoria. Os dados presentes no referido Relatório foram analisados e serão apresentados a seguir.

Além do texto base, foram utilizados os sites Bireme e PubMed, acrescidos dos serviços da Biblioteca J. Baeta Viana do Campus Saúde e da Biblioteca da Faculdade de Educação, ambas da Universidade Federal de Minas Gerais, e da Biblioteca da Faculdade de Ciências Médicas de Minas Gerais. As bases de dados consultadas foram: Medline, Lilacs, Scielo, Ovid, Prodoc e periódicos Capes. Deixar as siglas na $w e b$ foram encontradas referências da "Organização Internacional do Trabalho" (OIT) sobre o trabalho docente e suas implicações. As palavras-chave usadas isoladas e/ ou em cruzamentos foram: doenças dos professores (teachers' diseases), trabalho docente (teachers' work), doenças ocupacionais (occupational diseases), estresse e trabalho (stress and work), e burnout em professores (burnout among teachers).

Procedeu-se a uma análise das informações do Relatório à luz da literatura disponível sobre o assunto de modo a permitir a verificação de convergências, divergências e possíveis lacunas no conhecimento acumulado.

\section{Resultados e Discussão}

Os dados da Gerência de Saúde do Servidor e Perícia Médica (GSPM), reunidos no Relatório já mencionado, em Belo Horizonte, mostram freqüência importante de atendimentos médicos com solicitação para afastamento do trabalho por motivos de saúde (Belo Horizonte, 2003)

A análise dos dados mostra que a GSPM realizou 16.556 atendimentos de servidores da educação no período de maio de 2001 a abril de 2002. Infere-se, a partir disso, que as razões de procura da perícia médica não são banais, uma vez que, no universo citado, 92\% (15.243) dos atendimentos provocaram afastamento do trabalho. Os afastamentos no grupo geral de servidores são concentrados na categoria dos professores, totalizando 84\% dos servidores afastados (tabela 1).

\begin{tabular}{|c|c|c|}
\hline Funçāo & Freqüência & $\%$ \\
\hline Professor & 4463 & 84,2 \\
\hline Auxiliar de Escola & 438 & 8,3 \\
\hline Auxiliar Biblioteca Escolar & 87 & 1,6 \\
\hline Técnico Superior de Ensino & 142 & 2,7 \\
\hline Coordenador de Centro de Educação Infantil & 2 & 0,0 \\
\hline Secretário de Estabelecimento de Ensino & 40 & 0,8 \\
\hline Vice-diretor & 31 & 0,6 \\
\hline Diretor & 22 & 0,4 \\
\hline Supervisor de alimentação escolar & 6 & 0,1 \\
\hline Outros & 70 & 1,4 \\
\hline TOTAL & 5301 & 100,0 \\
\hline
\end{tabular}

Extraido e adaptado de: Prefeitura Municipal de Belo Horizonte/ Sindicato Único dos Trabalhadores do Ensino, 2003. Os dados apresentados nesta tabela referem-se aos afastamentos por cargos, nầo æe podend avaliar 0 numero real de docentes afastados.

Os dados de afastamento não podem expressar os problemas de saúde vividos pelos servidores, tampouco é possível estabelecer associações diretas desses problemas com o trabalho por eles desenvolvidos. Contudo, tais fatores são indicadores que nos permitem elaborar hipóteses articuladas aos dados da literatura citados ao longo deste artigo. Ou seja, os resultados das pesquisas isoladas, quando ana- 
lisados em conjunto, mostram coerência entre os seus achados e permitem a elaboração de hipóteses de pesquisa que visem a identificar associações do adoecimento com as características das escolas e as condições de trabalho vividas em suas dependências.

No período de maio de 2001 a abril de 2002 (tabela 2), os transtornos psíquicos ocuparam o primeiro lugar entre os diagnósticos que provocaram os afastamentos (15\%). 0 dado indica uma situação grave, e apesar de o Relatório não ter fornecido meios para distinguir os indivíduos, a freqüência do diagnóstico de transtornos psíquicos entre as causas de afastamento no trabalho é inquietante. Em segundo lugar, estão os afastamentos por doenças do aparelho respiratório (12\%) e, em terceiro, as doenças do sistema osteomuscular e do tecido conjuntivo (11\%). Vale registrar, contudo, que um mesmo professor pode ter sido afastado mais de uma vez.

Tabela 2: Grupamentos diagnósticos que levaram ao afastamento dos servidores da SMED, no período de maio de 2001 a abril de 2002.

\begin{tabular}{|c|c|c|}
\hline Diagnósticos & N. ${ }^{\circ}$ & $\%$ \\
\hline Transtornos mentais e comportamentais & 2333 & 15,3 \\
\hline Doenças do aparelho respiratório & 1855 & 12,2 \\
\hline Doenças do sistema osteomuscular e do tecido conjuntivo & 1754 & 11,5 \\
\hline Doenças do aparelho circulatório & 685 & 4,5 \\
\hline Doenças do sistema nervoso & 218 & 1,4 \\
\hline Doenças endócrinas, nutricionais e metabólicas & 153 & 1.0 \\
\hline Outros & 7394 & 48,5 \\
\hline Registros em branco & 851 & 5,6 \\
\hline Total & 15243 & 100,0 \\
\hline
\end{tabular}

Outros estudos obtiveram resultados semelhantes e encontraram associações com as condições de trabalho existentes. Zaragoza (1999) focalizou a evolução da saúde dos professores de 1982 a 1989, contabilizando as licenças médicas oficiais dos professores de ensino não universitários de Málaga e concluiu que, no período de sete anos, o número de professores em licença triplicou. Os diagnósticos mais freqüentes foram: distensões do tornozelo, laringites e depressões.

Shonfeld (1992) realizou um estudo longitudinal envolvendo 255 professoras recém- contratadas, na cidade de Nova York, com o objetivo de estimar os efeitos das condições de trabalho sobre sintomas depressivos no grupo alvo. Esse autor avaliou os sintomas depressivos pelo Center of Epidemilogic Studies - Depression Scale). 0 suporte social foi avaliado pelo Interpersonal Support Evaluation List. Para avaliar o grau de nocividade do ambiente escolar, foram desenvolvidos dois instrumentos: o Episodic Stressor Scale e o Strain Scale, distinguindo estressores eventuais de permanentes. Os resultados mostraram uma forte associação entre sintomas depressivos e ambientes de trabalho nocivos, bem como o surgimento precoce dos efeitos pesquisados, que se mantêm mesmo quando outros fatores de risco são controlados. Nesse estudo, o autor lembra também os resultados de sua pesquisa anterior, que associam ambientes perigosos, frustrantes ou carentes de controle a doenças depressivas.

Pitthers e Fogarty (1995) avaliaram o estresse e a tensão ocupacionais em professores utilizando o Occupational Stress Inventory, instrumento que avalia estresse ocupacional, sobrecarga acumulada e estratégias adotadas. Os maiores escores foram encontrados entre os professores, quando comparados com outros profissionais. Os resultados foram associados à sobrecarga de trabalho e aos conflitos com os superiores e as normas. Os autores ainda citam o estudo de Punch e Tuetteman, realizado em 1990, que avaliou 574 professores na Austrália e encontrou níveis de estresse psicológico duas vezes maior do que na população em geral. Estudos realizados nos EUA (1976), Austrália e Nova Zelândia (1982) e Reino Unido (1991), citados pelos mesmos autores, mostraram que um terço dos professores avaliados consideram seu trabalho "estressante" ou "muito estressante".

Um estudo quali-quantitativo, envolvendo 163 professores do ensino fundamental de Santa Maria (RS), investigou o estresse e os seus principais agentes desencadeadores, frente à inclusão de alunos com necessidades educacionais especiais. Nesse estudo, foram identifi- 
cados vários agentes estressores com os quais o professor tem de lidar no seu cotidiano profissional. A falta de preparo dos professores para o processo de inclusão foi a principal fonte geradora de estresse por eles apresentada. Os docentes pesquisados citam como fatores de agravamento do problema a quase inexistência de projetos de educação continuada que os capacite para enfrentar a "nova" demanda educacional; o elevado número de alunos por turmas; a infra-estrutura física inadequada; a falta de trabalhos pedagógicos em equipe; o desinteresse da família em acompanhar a trajetória escolar de seus filhos; a indisciplina cada vez maior; a desvalorização profissional e os baixos salários, situações que fogem de seu controle e preparo. Sentimentos de desilusão, de desencantamento com a profissão foram freqüentemente relatados, evidenciando a vulnerabilidade dos profissionais estudados ao estresse. A autora desse estudo conclui que as atividades pedagógicas permeadas por circunstâncias desfavoráveis forçam a uma reorganização e improvisação no trabalho planejado, distorcem o conteúdo das atividades e tornam o trabalho descaracterizado em relação às expectativas, gerando um processo de permanente insatisfação e induzindo a sentimentos de indignidade, fracasso, impotência, culpa e desejo de desistir, entre outros (Naujorks, 2002).

A investigação das condições de saúde e trabalho de professores da rede particular de ensino do estado da Bahia destaca uma grande proporção de adoecimento numa população relativamente jovem, com queixas importantes relacionadas ao funcionamento psíquico, como cansaço mental e nervosismo. Os resultados revelam uma freqüência de $20 \%$ de distúrbios psíquicos menores (DPM) aferido pelo Self Reporting Questionnaire (SRQ-20). Foram classificados como suspeitos de apresentar DPM os professores que responderam positivamente a sete ou mais questões dentre as vinte propostas apresentadas pelo teste. 0 referido grupo apresentou maior prevalência de problemas gerais de saúde, quando comparados ao grupo de professores sem DPM. No estudo, foram associados aos DPM os fatores que dizem respeito à organização do trabalho, tais como trabalho repetitivo, insatisfação no desempenho das atividades, ambiente intranqüilo e estressante, desgaste da relação professor-aluno, falta de autonomia no planejamento das atividades e ritmo acelerado de trabalho e pressão da direção (Araújo; Silvany-Neto, 1998).

Zaragoza (1999) chamou de mal-estar docente a sensação de mal-estar difuso e elaborou um modelo para explicar as relações funcionais existentes entre os múltiplos fatores indicadores do sintoma. Esse modelo considera que uma determinada combinação de fatores pode conduzir os professores a um estado de ansiedade, denominado esgotamento docente, que afeta sua personalidade. São mencionados os professores que souberam elaborar respostas efetivas e integradas ante o aumento de exigências e a enorme transformação a que se viu submetida a profissão docente. Às situações problemáticas que solicitam uma resposta do professor para reduzir o peso dos estímulos ameaçadores o autor chama de “tensão" e "estresse".

Ainda para Zaragoza, o absenteísmo seria um mecanismo de defesa utilizado contra a tensão derivada do exercício docente, uma forma de atingir um alivio para escapar das tensões acumuladas. Possivelmente, esse mecanismo está presente na população de educadores de Belo Horizonte afastados do trabalho por motivos de saúde no período estudado. $\mathrm{Na}$ população-alvo, do total dos afastamentos entre maio de 2002 a abril de 2003, 85\% referiamse à categoria dos professores (tabela 3). Como mencionado anteriormente, um mesmo professor pode ter sido afastado mais de uma vez.

Diversos estudos citados por Zaragoza (1999) buscam identificar os ciclos de estresse ao longo do ano escolar. Nos finais de trimestre (especialmente do primeiro) e no final do curso, o número de licenças médicas aumenta progressivamente. No início do primeiro trimestre, em especial, o número de licenças médicas aumenta bruscamente, detendo-se após feriados e tornan- 
do-se insignificante durante as férias de verão. $\mathrm{Na}$ pesquisa do autor, os problemas de saúde dos professores foram estudados exaustivamente no período de 1982 a 1984 e as causas de licença mais importantes foram os diagnósticos de traumatologia, geniturinários e obstétricos e os neuropsiquiátricos. Essas última causas, ainda que em terceiro lugar, constituíram o segundo lugar de importância pelo número de dias de licença que provocaram.

Tabela 3: Distribuição dos servidores afastados de suas atividades, por cargo, no período de maio de 2002 a abril de 2003.

\begin{tabular}{|l|l|l}
\hline \multicolumn{1}{|c|}{ Função } & Freqüência & $\%$ \\
\hline Professor & 4279 & 85,2 \\
\hline Auxiliar de Escola & 387 & 7,7 \\
\hline Auxiliar Biblioteca Escolar & 77 & 1,5 \\
\hline Técnico Superior de Ensino & 139 & 2,8 \\
\hline Coordenador de Centro de Educação Infantil & 2 & 0,0 \\
\hline Secretário de Estabelecimento de Ensino & 33 & 0,7 \\
\hline Vice-diretor & 29 & 0,6 \\
\hline Diretor & 13 & 0,3 \\
\hline Supervisor de alimentação escolar & 6 & 0,1 \\
\hline Outros TOTAL & 57 & 1,2 \\
\hline \multicolumn{1}{|c|}{} & $\mathbf{5 0 2 2}$ & $\mathbf{1 0 0 , 0}$ \\
\hline
\end{tabular}

Extraido e adaptado de: Prefeitura Municipal de Belo Horizonte/ Sindicato Único dos Trabalhadores do Ensino, 2003. Os dados apresentados nesta tabela referem-se aos afastamentos por cargos, nāo se podendo avaliar o número real de docentes afastados.

Diversos estudos realizados em Hong Kong nos últimos anos têm mostrado que ensinar é altamente estressante. Cerca de um terço dos professores pesquisados apresentavam sinais de estresse e burnout, entre os principais problemas de saúde. Observou-se distribuição heterogênea dos sintomas, sendo que alguns professores apresentaram sinais mais graves do que outros, variando de quadros leves de frustração, ansiedade e irritabilidade até o quadro de exaustão emocional, com sintomas psicossomáticos e depressivos severos (Chan, 2002).

Os dados disponíveis no Relatório da Prefeitura de Belo Horizonte, já mencionado, mantêm-se no período seguinte, entre maio de 2002 e abril de 2003. Os transtornos psíquicos continuam ocupando o primeiro lugar (tabela 4) entre os diagnósticos que provocaram o afas- tamento (16\%). As doenças do aparelho respiratório vêm em segundo lugar (12\%) e, as doenças do sistema osteomuscular e do tecido conjuntivo (10\%) permanecem em terceiro lugar entre os diagnósticos que provocaram o afastamento do trabalho.

Tabela 4: Grupamentos diagnósticos que levaram ao afastamento dos servidores da SMED, no período de maio de 2002 a abril de 2003

\begin{tabular}{|l|c|c|}
\hline \multicolumn{1}{|c|}{ Diagnósticos } & N. $^{\circ}$ & $\%$ \\
\hline Transtornos mentais e comportamentais & 2247 & 16,3 \\
\hline Doenças do aparelho respiratório & 1611 & 11,7 \\
\hline Doenças do sistema osteomuscular e do tecido conjuntivo & 1441 & 10,5 \\
\hline Doenças do aparelho circulatório & 610 & 4,4 \\
\hline Doenças do sistema nervoso & 210 & 1,5 \\
\hline Doenças endócrinas, nutricionais e metabólicas & 141 & 1.0 \\
\hline Outros & 6665 & 48,4 \\
\hline Registros em branco Total & 848 & 6,2 \\
\hline \multicolumn{1}{|c|}{} & $\mathbf{1 3 7 7 3}$ & $\mathbf{1 0 0 , 0}$ \\
\hline $\begin{array}{l}\text { Extraído e adaptado de: Prefeltura Municipal de Belo Horizonte/ Sindicato Unico dos } \\
\text { Trabalhadores do Ensino, 2003. }\end{array}$ & \multicolumn{2}{c}{} \\
\hline
\end{tabular}

0 estudo de Siqueira e Ferreira (2003) encontrou resultados semelhantes. Com o objetivo de investigar o absenteísmo docente no ensino fundamental, as autoras estudaram as professoras das séries iniciais da rede pública de ensino da cidade de Florianópolis (SC) por meio da análise dos prontuários dos docentes que se afastaram do trabalho para tratamento de saúde. Constatou-se que as causas mais freqüentes geradoras desses afastamentos foram, em ordem decrescente: as doenças do aparelho respiratório, os problemas do aparelho locomotor, os problemas de saúde na família e problemas psicológicos e/ ou psiquiátricos. Embora no estudo citado os transtornos psíquicos ocupem o quarto lugar nas causas de afastamento do trabalho em docentes, ainda assim se encontram entre os mais prevalentes, bem como os problemas respiratórios e do aparelho locomotor.

Neto et al. (2000) estudaram o perfil de professores de 58 escolas da rede particular de ensino de Salvador (Bahia). Os resultados de seus estudos descrevem uma população cuja média de idade é de 35 anos, mulheres (75\%) e casados (56\%) em sua maioria. Chama atenção o duplo vínculo de trabalho com escola pública (20\%), sendo o número médio de aulas por se- 
mana de 25 horas. Entre as queixas de saúde foram citadas: dor na garganta, dor nas pernas e costas, rouquidão e cansaço mental. As características do trabalho associadas às queixas foram: salas inadequadas, trabalho repetitivo, exposição ao pó de giz, ambiente de trabalho estressante, ritmo acelerado de trabalho, desempenho das atividades sem materiais e equipamentos adequados e posição de trabalho incômoda. Ressaltam-se os aspectos positivos do trabalho mencionados pelos professores, que destacam a boa relação com os colegas, a autonomia de planejamento, a satisfação no desempenho das atividades e a existência de banheiro privativo e existência de espaço para descanso.

Delcor et al. (2004) realizaram um estudo transversal na rede particular de ensino da cidade de Vitória da Conquista (Bahia) no grupo de professores do pré-escolar ao ensino médio. Foi utilizado um formulário auto-aplicado com cinco blocos de questões com informações sobre as características sociodemográficas, econômicas e ocupacionais e sobre a saúde física e mental dos docentes. Os resultados apontam uma população jovem (idade média de 34,5 anos), composta, principalmente, de mulheres (83\%), em sua maioria casadas (65\%), com nível de escolaridade superior (72\%), sendo que mais da metade (52\%) trabalhava em outra escola. Entre as queixas de saúde apresentadas destacaram-se: cansaço mental (60\%), dor nos braços e ombros (52\%), dor nas costas (51\%), formigamento nas pernas $(47 \%)$, dor na garganta $(46 \%)$ e rouquidão (60\%). Permanecer em pé e corrigir trabalhos escolares foram os esforços físicos mais freqüentes. 0 Job Content Questionnaire, incorporado ao questionário, identificou como aspectos negativos para o bom desenvolvimento do trabalho o ritmo acelerado (68\%), posição inadequada ou incômoda do corpo (65\%), atividade física rápida e contínua (64\%) e longos períodos de concentração numa mesma tarefa (52\%). Mais de 90\% dos professores que participaram do estudo demonstraram necessidade de maior criatividade e de maior nível de habilidade para aprender coisas novas. Segundo a população estudada, os colegas de trabalho foram considerados "amigáveis”. O Self Questionnaire Report-20 estimou uma prevalência de distúrbios psíquicos menores em $41,5 \%$, variando de 17 a 66\%, a depender da escola onde o profissional se inseria, estando fortemente associada ao trabalho repetitivo, ao volume excessivo e ao ritmo acelerado de trabalho, à intensa concentração em uma mesma tarefa e tempo insuficiente para a realização de outras.

Em Campinas, estudo realizado entre os professores do ensino fundamental da rede particular no ano de 2000 coloca em evidência a contaminação do tempo extratrabalho, sendo freqüentes as dificuldades em realizar as tarefas diárias com satisfação explicando o quadro encontrado de nervosismo, tensão e preocupação (Oliveira, 2001).

Codo (1999) estudou uma amostra de quase 39 mil trabalhadores em educação em todo o país e identificou que 32\% dos indivíduos apresentavam baixo envolvimento emocional com a tarefa, $25 \%$ se encontravam com exaustão emocional e 11\% com quadro de despersonalização, podendo-se dizer, em termos práticos, que 48\% da população estudada apresentava burnout. 0 autor esclarece os elementos que podem estar associados às queixas e ao adoecimento. Lembra que o trabalho do professor não se restringe ao exercício de sua função dentro da sala de aula, exige atualização e preparação constantes para ser realizado de modo satisfatório. Muitas tarefas são realizadas sem a presença dos alunos, fora da sala de aula e, freqüentemente, fora da escola, estendendo a jornada de trabalho. Quando o professor ministra aulas em várias turmas para alunos em níveis de ensino, escolas e turnos diferentes, a preparação das aulas vai requerer avaliações múltiplas e esquemas variados. Serão necessários maior investimento de tempo na execução de um volume maior de trabalho e mais dedicação e esforço intelectual.

É Codo ainda que enfatiza o maior investimento emocional, na medida em que diariamente são estabelecidos vínculos com os alunos, com outros professores e funcionários 
da escola. No conjunto, os fatores citados explicariam a sobrecarga mental, situação que culmina com a exaustão mental, em que o professor se sente exaurido emocionalmente e o trabalho perde o sentido. As situações mais freqüentemente vividas, geradas pelo sofrimento no trabalho, são: depressão, fadiga, insatisfação, frustração, medo, angústia e ansiedade, até chegar à exaustão.

A pesquisa de Noronha (2001) também identificou os sentimentos de insatisfação, frustração e ansiedade relatados nas entrevistas evidenciando a associação entre as queixas de cansaço e sobreesforço físico e mental solicitados pelo conjunto das atividades estudadas por meio das técnicas da escola francesa de ergonomia.

0 estudo ergonômico realizado por Gomes (2002) em um grupo de onze professores (sete mulheres e quatro homens), com idade entre 26 e 60 anos, em uma escola estadual do Rio de Janeiro, com 283 professores e cinco mil alunos, sendo 40 a 45 alunos por turma, divididos em três turnos, evidenciaram: insatisfação por trabalhar em mais de uma escola; referência à sobrecarga de trabalho determinada por diferentes fatores (gestão, política, infra-estrutura e tempo). A autora menciona a invasão do tempo e do espaço extra-escolar pelo trabalho, a intensificação das atividades no final do ano letivo e o número excessivo de alunos por turno. Finalmente, o estudo citado aborda os efeitos da situação diagnosticada, refletindo-se em agitação, estresse e irritação do professor, que se sente responsável pela formação ética e moral dos alunos. Os problemas de saúde identificados foram: sensação de intenso mal-estar generalizado; ansiedade, tensão, nervosismo, irritabilidade, depressão, angústia e esgotamento; perturbações do sono; problemas digestivos; problemas respiratórios e da voz. Os professores estudados expressaram frustrações diante da precariedade de recursos materiais que dificulta o cumprimento de objetivos planejados. 0 cenário da escola deixa pouca margem para a criatividade e autonomia do professor face às normas educacionais vigentes, assim como a obrigatoriedade de formação específica em cursos estipulados pelo seu gestor e, também, prescrição do tipo de avaliação dos alunos.

\section{Conclusão}

Os dados e as conclusões dos estudos interessados em descrever o perfil de adoecimento dos professores são convergentes, independentemente da população e da região estudada. Observou-se que os professores têm mais risco de sofrimento psíquico de diferenciados matizes e a prevalência de transtornos psíquicos menores é maior entre eles, quando comparados a outros grupos.

Embora os dados acerca de afastamentos por licenças médicas não indiquem a real dimensão do problema de saúde de uma categoria de trabalhadores, os indicadores podem ser tomados como pistas sobre situações que merecem maior aprofundamento e análise. No caso estudado, os dados da literatura são coerentes com os registros na Gerência de Saúde do Servidor e Perícia Médica, em Belo Horizonte, apresentados anteriormente, os quais colocam em evidência a prevalência de afastamentos dos professores, sendo os transtornos psíquicos responsáveis pelo maior número de casos.

0 acúmulo de conhecimentos no campo de estudo das relações saúde e trabalho permite supor associações entre os problemas de saúde identificados na categoria dos professores e as condições ergonômicas de trabalho também descritas na literatura consultada.

Seriam úteis desenhos de estudos voltados para compreender a inadequação entre as mudanças educacionais propostas e implementadas, e a realidade que os trabalhadores enfrentam nas escolas. As contradições existentes podem estar na origem da exposição aos fatores de risco para o adoecimento da categoria dos trabalhadores do ensino. 


\section{Referências bibliográficas}

ARAÚJO, T. M.; SILVANY-NETO, A. M. (Orgs.). Condições Trabalho e Saúde dos Professores da Rede Particular de Ensino. Sindicato dos Professores no Estado da Bahia/ Universidade Federal da Bahia/ Confederação Nacional dos Trabalhadores em Estabelecimentos de Ensino, Salvador, set. 1998, pp. 5-42.

BARRETO, R. G.; LEHER, R. Trabalho docente e as reformas neoliberais. In: OLIVEIRA, D. A. Reformas educacionais na América Latina e os trabalhadores docentes. Belo Horizonte: Autêntica, 2003. p. 39-60.

CHAN, D. W. Hardiness and its role in the stress-burnout relationship among prospective Chinese teachers in Hong Kong. Teaching and Teacher Education, v. 19, p. 381-395, 2003.

CODO, W. (Org.). Educação: carinho e trabalho. Petrópolis: Vozes, 1999.

DELCOR, N., S. et al. Condições de trabalho e saúde dos professores da rede particular de ensino de Vitória da conquista, Bahia, Brasil. Cadernos de Saúde Pública, Rio de Janeiro, v.20, n.1, p. 187-196, jan.-fev. 2004.

GOMES, L. Trabalho multifacetado de professores/as: a saúde entre limites. Dissertação (Mestrado) - Escola Nacional de Saúde, Fundação Osvaldo Cruz, Rio de Janeiro, 2002.

MINISTÉRIO DA EDUCAÇÃO E CULTURA. 0 déficit de professores no país. Disponível em: portaldoprofessor.inep.gov.br/ estatisticas.jsp.

NAUJORKS, M. I. Stress e Inclusão: indicadores de stress em professores frente a inclusão de alunos com necessidades educacionais especiais. Cadernos de Educação Especial. Universidade Federal de Santa Maria. Centro de Educação/ Departamento de educação Especial/ Laboratório de Pesquisa e Documentação (Lapedoc), v. 1, n. 20, 2002.

NETO, A. M. S. et al. Condições de trabalho e saúde de professores da rede particular de ensino de Salvador, Bahia. Revista Bahiana de Saúde Pública, v. 24. n. 1/2, p. 42-56, jan/dez. 2000.

NORONHA, M. M. B. Condições do exercício profissional da professora e dos possíveis efeitos sobre a saúde: estudo de casos das professoras do ensino fundamental em uma escola pública de Montes Claros, Minas Gerais. Dissertação (Mestrado) Faculdade de Medicina da UFMG. Belo Horizonte, 2001. 157 p.

OLIVEIRA, D. A. As reformas educacionais e suas repercussões sobre o trabalho docente. In América Latina e os trabalhadores docentes. Autêntica: Belo Horizonte, 2003, p. 13-35.

Reformas educacionais na

OLIVEIRA, M. G. Condições de trabalho, gênero e saúde: sofrimento e estresse. um estudo de caso com os profissionais docentes do ensino superior privado de Belo Horizonte. Dissertação (Mestrado) - Pontifícia Universidade Católica de Minas Gerais, Belo Horizonte, 2001.

ORGANIZAÇÃO INTERNACIONAL DO TRABALHO. A condição dos professores: recomendação Internacional de 1966, um instrumento para a melhoria da condição dos professores. Genebra: OIT/ Unesco, 1984.

PITHERS, R. T.; FOGARTY, G. J. Symposium on teacher stress: occupational stress among vocational teachers. British Journal of Educational Psychology, v. 65, p. 3-14, 1995.

SCHONFELD, I. S. A longitudinal study of occupational stressors and depressive symptoms in first-year female teachers. Teaching \& teacher education, v. 8, n.2, p. 151-158, 1992

SIQUEIRA, M. J. T.; FERREIRA, E. S. Saúde das professoras das séries iniciais; 0 que 0 gênero tem a ver com isso? Psicologia, Ciência e Profissão, v. 23, n. 3, p. 76-83, 2003.

SOUZA, K. R. et al. Trajetória do Sindicato Estadual dos Profissionais da Educação do Rio de Janeiro (SEPE-RJ) na luta pela saúde no trabalho. Ciência e Saúde Coletiva, v. 8, n. 4, p. 1057-1068, 2003. 
TEIXEIRA, L. H. G. Políticas públicas de educação e mudança nas escolas: um estudo da cultura escolar. In: OLIVEIRA, D. A.; DUARTE, M. R. T. (Orgs.) Política e trabalho na escola: administração dos sistemas de educação básica. 2.ed., Belo Horizonte, 2001, p. 177-190.

ZARAGOZA, J. M. E. 0 mal-estar docente: a sala de aula e a saúde dos professores. Tradução: Durley de Carvalho Cavicchia. $3^{\mathrm{a}}$ ed., Bauru: Edusc, 1999.

Recebido em 03.08.04

Modificado em 06.12.04

Aprovado em 07.04.05

Sandra Maria Gasparini é mestre em Saúde Pública pelo Programa de Pós-Graduação em Saúde Pública da Faculdade de Medicina da UFMG. Membro do Grupo de Estudos sobre Trabalho Docente [GESTRAD0] da Faculdade de Educação da Universidade Federal de Minas Gerais, apoiado pelo CNPq e FAPEMIG.

Sandhi Maria Barreto é professora do Programa de Pós-Graduação em Saúde Pública da Faculdade de Medicina da UFMG da Universidade Federal de Minas Gerais.

Ada Ávila Assunção é professora do Programa de Pós-Graduação em Saúde Pública da Faculdade de Medicina da Universidade Federal de Minas Gerais. Membro do Grupo de Estudos sobre Trabalho Docente [GESTRAD0] da Faculdade de Educação da Universidade Federal de Minas Gerais, apoiado pelo CNPq e FAPEMIG. 